SFB

A law of large numbers for the power variation of fractional

823

Lévy processes

Sven Glaser

Nr. 31/2013

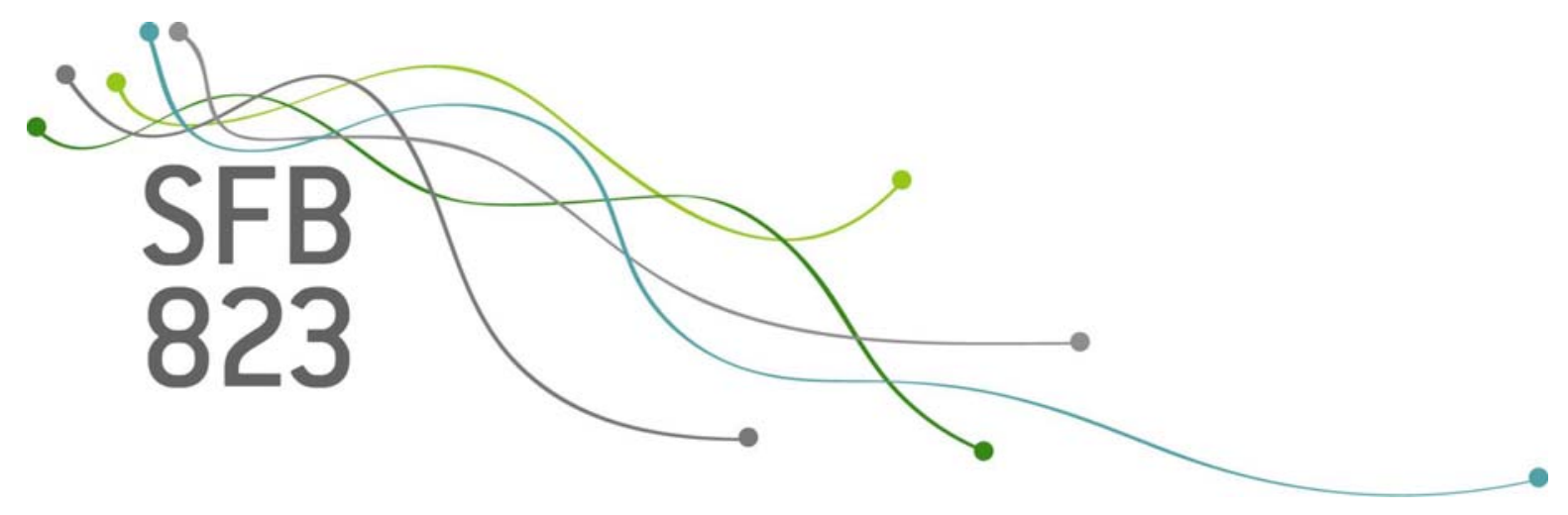





\title{
A LAW OF LARGE NUMBERS FOR THE POWER VARIATION OF FRACTIONAL LÉVY PROCESSES
}

\author{
SVEN GLASER
}

\begin{abstract}
We prove a law of large numbers for the power variation of an integrated fractional process in a pure jump model. This yields consistency of an estimator for the integrated volatility where we are no longer restricted to a Gaussian model.
\end{abstract}

\section{INTRODUCTION}

In this article we develop a law of large numbers for the realized power variation of integrated fractional Lévy processes. The realized power variation was introduced by Barndorff-Nielsen and Shephard [BNS02, BNS03, BNS04a, BNS04b] in the context of stochastic volatility models to estimate the integrated volatility. Integrated volatility quantifies the level of volatility which is important for pricing and risk assessment. [CNW06] developed limit theorems for the power variation of integrated fractional processes of the form

$$
Z_{t}:=\int_{0}^{t} u_{s} d B_{s}^{H}
$$

where $B^{H}$ denotes a fractional Brownian motion with Hurst parameter $H \in(0,1)$. We now want to go one step further and replace the fractional Brownian motion in the representation of the process $\mathrm{Z}$ above by a fractional Lévy process $L^{H}$ satisfying some properties which are given later. The biggest advantage is that marginal distributions are no longer restricted to be normal but taken from the class of infinitely divisible distributions which might possess heavier tails than a normal distribution. Another interesting question in the framework of fractional processes is to estimate the so called Hurst-parameter which provides information about the dependence structure, cf. [BCI04].

We define fractional Lévy processes by replacing the Brownian motion in the moving average representation of fractional Brownian motion, that is,

$$
B_{t}^{H}=C \int_{-\infty}^{\infty} a\left((t-s)_{+}^{H-1 / 2}-(-s)_{+}^{H-1 / 2}\right)+b\left((t-s)_{-}^{H-1 / 2}-(-s)_{-}^{H-1 / 2}\right) d B_{s}
$$

2010 Mathematics Subject Classification. 60F99, 60G22, 62H12.

Key words and phrases. fractional Lévy processes, limit theorems, estimation of the integrated volatility, infinitely divisible distributions, power variation. 
S. GLASER

where $a, b$ and $C$ are real valued constants, by a two sided Lévy process $L$. For simplicity we consider the case $a=1$ and $b=0$. By linearity of the integral and symmetry of the integrand the proofs are the same as in the general case. We define

$$
X_{t}^{\gamma}:=\int_{-\infty}^{\infty}(t-s)_{+}^{\gamma}-(-s)_{+}^{\gamma} d L_{s}, \quad t \in \mathbb{R},
$$

as fractional Lévy process, where we also replaced the exponent $H-\frac{1}{2}$ in the definition of fractional Brownian motions by $\gamma$. [EW13] yields a good overview about the properties of fractional Lévy processes. In Corollaries 2, 3 and 4 of [EW13] it was shown under which conditions processes of this kind are well defined. Sections 3 and 4 of [EW13] provide distributional and path properties of those processes.

This paper is structured as follows: in the next section we will give an introduction to fractional Lévy processes. Also we provide the results we use in the proof of our main result. Section 3 provides the main result of this article, that is convergence theorem for the power variation of integrated fractional Lévy processes.

\section{UNDERLYING DEFINITIONS AND NOTATIONS}

We start with the definition of fractional Lévy processes. Therefore let $\left(L_{t}\right)_{t>0}$ be a real valued Lévy process on a probability space $(\Omega, \mathcal{F}, \mathbb{P})$ with characteristic triplet $(b, 0, v)$, so the Lévy Khintchine formula reduces to

$$
\mathbb{E}\left[e^{i u X_{t}}\right]=\exp \left(i b u t+t \int_{\mathbb{R}}\left(e^{i u x}-1-i u x \mathbb{1}_{|x| \leq 1}(x)\right) d v(x)\right) .
$$

Note that we consider Lévy processes which do not have a Brownian component. This is sufficient since if we had a Brownian component, we could decompose our underlying Lévy process into the Brownian part and a pure jump Lévy process which we are considering here. Then the integral of a kernel function decomposes into two parts, a fractional Brownian motion and a fractional Lévy process of the kind we are considering here.

Fractional Lévy processes are defined as integrals of deterministic kernels with respect to twosided Lévy processes, this is $\left(L_{t}\right)_{t \in \mathbb{R}}$ with $L_{t}=L_{t}^{1}$ for $t \geq 0$ and $L_{t}=-L_{-t^{-}}^{2}$ for $t<0$, where $L^{1}$ and $L^{2}$ are two independent copies of the process $L$ above. As mentioned in the introduction, we will restrict ourselves to the case where the kernel function is given by

$$
f_{\gamma}^{+}(t, s):=(t-s)_{+}^{\gamma}-(-s)_{+}^{\gamma} \text {. }
$$

There are other possibilities, namely

- $f_{\gamma}^{-}(t, s):=\left((t-s)_{-}^{\gamma}-(-s)_{-}^{\gamma}\right)$,

- $f_{\gamma}(t, s):=a f_{\gamma}^{+}(t, s)+b f_{\gamma}^{-}(t, s)$,

but these kernels go analogously to our case and will be omitted. We also state an integrability condition for the function $f_{\gamma}^{+}(t, s)$ :

Lemma 1. The function $\left|f_{\gamma}^{+}(t, s)\right|^{\delta}$ is integrable at 0 and $t$ iff either $\gamma>0$ or both of $\gamma<0$ and $\delta<\frac{1}{-\gamma}$ are satisfied. It is integrable at $-\infty$ iff $\delta>\frac{1}{1-\gamma}$. 
Proof. See [EW13], Proposition 2.

Now we can define fractional Lévy processes:

Definition 1. Let $L$ be a two sided Lévy process as above and $\gamma \in \mathbb{R}$ such that the integral $\int_{\mathbb{R}} f_{\gamma}^{+}(t, s) d L_{s}$ exists for all $t \in \mathbb{R}$. Then the stochastic process, defined by

$$
X_{t}^{\gamma}:=\int_{\mathbb{R}} f_{\gamma}^{+}(t, s) d L_{s}, \quad t \in \mathbb{R}
$$

is called fractional Lévy process.

Remark 1. The existence of the integral $\int_{\mathbb{R}} f_{\gamma}^{+}(t, s) d L_{s}$ depends on the driving Lévy process $L$ and the parameter $\gamma$. [EW13] provides an existence result of fractional Lévy processes where the drift is $b=\int_{|x| \leq 1} x d v(x)$ or $b=-\int_{|x|>1} x d v(x)$ depending on the existence of the first moment of the Lévy measure $v$.

Since we only consider fractional Lévy processes which are local self similar (cf. Proposition 2 below) we can omit a section about the existence of the integral and give the statements in that case.

In the following we also need the characteristic function of fractional Lévy processes:

Proposition 1. Let $L$ have characteristic triplet $(b, 0, v)$ with $b \in \mathbb{R}$, given by $b=\int_{|x| \leq 1} x d v$ respectively $b=-\int_{|x|>1} x d v$, depending on the driving Lévy process and let $\gamma$ be as in the definition before. Then the fractional Lévy process $X_{t}^{\gamma}$, defined in Definition 1, has stationary increments. Moreover, for $m \in \mathbb{N}, t_{1}, \ldots, t_{m} \in \mathbb{R}$ and $u_{1}, \ldots, u_{m} \in \mathbb{R}$ its finite dimensional distributions exhibit the characteristic function given by

$$
\mathbb{E}\left[\exp \left\{i \sum_{j=1}^{m} u_{j} X_{t_{j}}^{\gamma}\right\}\right]=\exp \left\{\int_{\mathbb{R}} \psi_{\gamma}\left(\sum_{j=1}^{m} u_{j} f_{\gamma}^{+}\left(t_{j}, s\right)\right) d s\right\},
$$

where

$$
\psi_{\gamma}(y)=i y b+\int_{\mathbb{R}}\left(e^{i x y}-1-i x y \mathbb{1}_{|x| \leq 1}(x)\right) d v(x), \quad y \in \mathbb{R} .
$$

In particular, the distribution of $X_{t}^{\gamma}$ is infinitely divisible for all $t \in \mathbb{R}$.

Proof. The proof is e.g. done in [EW13] Proposition 4.

The next example is the same as in [EW13], example 1.

Example 1. Introduced as an extension of fractional Brownian motions the so-called linear fractional stable motion is one example of a fractional Lévy process. For $0<\alpha<2$ it was introduced in Samorodnitsky and Taqqu ([ST00], Example 3.6.5 and Section 7.4) as the process

$$
Y_{t}^{\alpha, H}=\int_{\mathbb{R}} f_{H-\frac{1}{\alpha}}(t, s) d M_{s}, \quad t \in \mathbb{R},
$$

where $M$ is an $\alpha$-stable random measure with Lebesgue control measure, $a, b$ are real constants with $|a|+|b|>0$, and with $0<H<1, H \neq \frac{1}{\alpha}$. By Lemma 1 it is $\int_{-\infty}^{\infty}\left|f_{\gamma}(t, s)\right|^{\alpha} d s<\infty$ so 
S. GLASER

the process is well defined. Here we also consider the case $a=1$ and $b=0$ and in addition to that the symmetric case, this is if the skewness intensity equals 0 . Then these processes arise as a special case of our definition of fractional Lévy processes. To see this we define a Lévy measure by $\frac{d v(x)}{d x}=\frac{c}{|x|^{1+\alpha}}$ and denote by $L^{\alpha}$ the corresponding Lévy process. Then we can define the fractional Lévy process

$$
X_{t}=\int_{\mathbb{R}} f_{\gamma}(t, s) d L_{s}^{\alpha}, \quad t \in \mathbb{R} .
$$

This process exists for $\gamma \in\left(-\frac{1}{\alpha}, 1-\frac{1}{\alpha}\right)$ (see remark below). In the proof of Proposition 2 we see that for $\gamma=H-\frac{1}{\alpha}$ the processes $Y^{\alpha, H}$ and $X$ are equal in distribution.

Remark 2. The existence of the process $X$ for the parameter $\gamma \in\left(-\frac{1}{\alpha}, 1-\frac{1}{\alpha}\right)$ in the example above is a direct consequence of corollaries 2, 3 and 4 of [EW13]. As mentioned above, the drift $b$ depends on the existence of the first moment. In this example the drift $b$ is arbitrary in the case $\alpha=1$, for $0<\alpha<1$ it is $b=\int_{|x| \leq 1} x d v$ and for $1<\alpha<2$ it is $b=-\int_{|x|>1} x d v$.

The next proposition shows the local self similarity of fractional Lévy processes. The proposition is essentially the same as Theorem 4.7 in [Mar06]. The difference is that we keep the representation as an increment which is important for our proofs later.

Proposition 2. Let $\alpha \in(0,2)$ and L a Lévy process whose Lévy measure $v$ has a Lebesgue-density $g$ such that

(1) $g(x)=\mathcal{O}\left(|x|^{-1-\alpha}\right)$ for $x \rightarrow 0$,

(2) $g(x) \leq C|x|^{-1-\alpha}, \quad \forall x \in \mathbb{R}$

where $C>0$, and an appropriate drift $b$ (depending on $\alpha$, cf. Remark 2$)$. For $\gamma \in\left(-\frac{1}{\alpha}, 1-\frac{1}{\alpha}\right)$, the process $L_{t}^{H}=\int_{\mathbb{R}} f_{\gamma}^{+}(t, s) d L_{s}$ exists for all $t \in \mathbb{R}$ and is locally self similar with parameter $H=\gamma+1 / \alpha$, i.e. for each $a \in \mathbb{R}$, it holds

$$
\lim _{\varepsilon \searrow 0}\left(\varepsilon^{-H}\left(L_{\varepsilon(t+a)}^{H}-L_{\varepsilon t}^{H}\right)\right)_{t \in \mathbb{R}} \stackrel{d}{=}\left(X_{t+a}-X_{t}\right)_{t \in \mathbb{R}}
$$

where the limit is in distribution for all finite dimensional margins and the process $X$ is the linear fractional stable motion with parameter $\alpha$ as in Example 1.

Proof. The proof is similar to the one in [Mar06]. The existence of the process $L^{H}$ follows by the second property of the Lebesgue-density $g$ and the existence of the process $X$ we discussed in the previous remark. The proof is done in two steps, first, we calculate the limit of characteristic functions, then we show that this is indeed the characteristic function of the linear fractional stable motion we introduced in Example 1. 
Starting with the first step let $u_{1}, \ldots, u_{m} \in \mathbb{R},-\infty<t_{1}<\cdots<t_{m}<\infty \in \mathbb{R}$ and $m \in \mathbb{N}$. Then we calculate:

$$
\begin{aligned}
& \log \mathbb{E} {\left[\exp \left\{i \sum_{k=1}^{m} u_{k} \varepsilon^{-H}\left(L_{\varepsilon\left(t_{k}+a\right)}^{H}-L_{\varepsilon t_{k}}^{H}\right)\right\}\right] } \\
&=\int_{\mathbb{R}} \int_{\mathbb{R}}\left(e^{i x \sum_{k=1}^{m} u_{k} \varepsilon^{-H}\left(\left(\varepsilon\left(t_{k}+a\right)-s\right)_{+}^{\gamma}-\left(\varepsilon t_{k}-s\right)_{+}^{\gamma}\right)}-1\right. \\
&\left.-i x \sum_{k=1}^{m} u_{k} \varepsilon^{-H}\left(\left(\varepsilon\left(t_{k}+a\right)-s\right)_{+}^{\gamma}-\left(\varepsilon t_{k}-s\right)_{+}^{\gamma}\right) \mathbb{1}_{|x| \leq 1}\right) d v(x) d s \\
&=\int_{\mathbb{R}} \int_{\mathbb{R}}\left(e^{i x \sum_{k=1}^{m} u_{k} \varepsilon^{-1 / \alpha}\left(\left(t_{k}+a-s\right)_{+}^{\gamma}-\left(t_{k}-s\right)_{+}^{\gamma}\right)}-1\right. \\
&\left.-i x \sum_{k=1}^{m} u_{k} \varepsilon^{-1 / \alpha}\left(\left(t_{k}+a-s\right)_{+}^{\gamma}-\left(t_{k}-s\right)_{+}^{\gamma}\right) \mathbb{1}_{|x| \leq 1}\right) \varepsilon d v(x) d s,
\end{aligned}
$$

where the last equation results by substituting $s$ by $s \varepsilon$. We will now substitute $x=\varepsilon^{1 / \alpha} y$ and since $\int_{1 \leq|y| \leq \varepsilon^{-1 / \alpha}} i \frac{y}{|y|^{1+p}} \sum_{k=1}^{m} u_{k}\left(\left(t_{k}+a-s\right)_{+}^{\gamma}-\left(t_{k}-s\right)_{+}^{\gamma}\right) d y=0$ we obtain

$$
\begin{aligned}
& \log \mathbb{E} {\left[\exp \left\{i \sum_{k=1}^{m} u_{k} \varepsilon^{-H}\left(L_{\varepsilon\left(t_{k}+a\right)}^{H}-L_{\varepsilon\left(t_{k}+a\right)}^{H}\right)\right\}\right] } \\
&=\int_{\mathbb{R}} \int_{\mathbb{R}}\left(e^{i y \sum_{k=1}^{m} u_{k}\left(\left(t_{k}+a-s\right)_{+}^{\gamma}-\left(t_{k}-s\right)_{+}^{\gamma}\right)}-1\right. \\
&\left.-i y \sum_{k=1}^{m} u_{k}\left(\left(t_{k}+a-s\right)_{+}^{\gamma}-\left(t_{k}-s\right)_{+}^{\gamma}\right) \mathbb{1}_{\left|y \varepsilon^{1 / \alpha}\right| \leq 1}\right) \varepsilon d v\left(y \varepsilon^{1 / \alpha}\right) d s \\
&=\int_{\mathbb{R}} \int_{\mathbb{R}}\left(e^{i y \sum_{k=1}^{m} u_{k}\left(\left(t_{k}+a-s\right)_{+}^{\gamma}-\left(t_{k}-s\right)_{+}^{\gamma}\right)}-1\right. \\
&\left.\quad-i y \sum_{k=1}^{m} u_{k}\left(\left(t_{k}+a-s\right)_{+}^{\gamma}-\left(t_{k}-s\right)_{+}^{\gamma}\right) \mathbb{1}_{|y| \leq 1}\right) \varepsilon d v\left(y \varepsilon^{1 / \alpha}\right) d s \\
&=: \int_{\mathbb{R}} \int_{\mathbb{R}} F(y, s) \varepsilon d v\left(y \varepsilon^{1 / \alpha}\right) d s .
\end{aligned}
$$

We now use the asymptotic behaviour of the Lévy measure:

$$
\varepsilon d v\left(y \varepsilon^{1 / \alpha}\right)=\varepsilon g\left(y \varepsilon^{1 / \alpha}\right) \varepsilon^{1 / \alpha} d y \stackrel{\varepsilon}{\sim}{ }^{\text {small }} \varepsilon^{1+1 / \alpha}\left|\varepsilon^{1 / \alpha} y\right|^{-1-\alpha} d y=|y|^{-1-\alpha} d y,
$$


which is the Lévy measure of the process $X$ introduced in Example 1. If we now pass to the limit for $\varepsilon \rightarrow 0$ we can conclude

$$
\log \mathbb{E}\left[\exp \left\{i \sum_{k=1}^{m} u_{k} \varepsilon^{-H}\left(L_{\varepsilon\left(t_{k}+a\right)}^{H}-L_{\varepsilon\left(t_{k}+a\right)}^{H}\right)\right\}\right] \rightarrow \int_{\mathbb{R}} \int_{\mathbb{R}} F(y, s) \frac{d y}{|y|^{1+\alpha}} d s .
$$

This is exactly the representation in Proposition 1 of characteristic functions of fractional Lévy processes, in particular this is the representation of the characteristic function of the process $X$.

To prove the second step we use Euler-representation of the exponential function. For the sake of simplicity we define $z_{s}:=\sum_{k=1}^{m} u_{k}\left(\left(t_{k}+a-s\right)_{+}^{\gamma}-\left(t_{k}-s\right)_{+}^{\gamma}\right)$ and use the symmetry of the sine function to calculate

$$
\begin{aligned}
& \int_{\mathbb{R}} i\left(\sin y z_{s}-y z_{s} \mathbb{1}_{|y| \leq 1}\right) \frac{d y}{|y|^{1+\alpha}} d s \\
= & i \int_{-1}^{1}\left(\sin y z_{s}-y z_{s}\right) \frac{d y}{|y|^{1+\alpha}}+i \int_{|y|>1} \sin y z_{s} \frac{d y}{|y|^{1+\alpha}} \\
= & 0
\end{aligned}
$$

where all those integrals exist. We now use this fact to conclude

$$
\begin{aligned}
& \int_{\mathbb{R}} \int_{\mathbb{R}} F(y, s) \frac{d y}{|y|^{1+\alpha}} d s \\
= & 2 \int_{\mathbb{R}} \int_{0}^{\infty}\left(\cos \left(y z_{s}\right)-1\right) \frac{d y}{|y|^{1+\alpha}} d s \\
\stackrel{(*)}{=} & 2 \int_{\mathbb{R}} \int_{0}^{\infty}(\cos (x)-1) \operatorname{sign}\left(z_{s}\right)\left|z_{s}\right|^{\alpha} \frac{d x}{|x|^{1+\alpha}} d s \\
= & 2 \int_{0}^{\infty}(\cos (x)-1) \frac{d x}{|x|^{1+\alpha}} \int_{\mathbb{R}} \operatorname{sign}\left(z_{s}\right)\left|z_{s}\right|^{\alpha} d s,
\end{aligned}
$$

which is the characteristic function of the linear fractional stable motion, see [ST00, p.114]. Equation (*) holds by substituting $x=y z_{s}$. This proves the equality in distribution of the processes $X$ and $Y^{\alpha, H}$ in Example 1.

From now on we will only consider fractional Lévy processes which are local self similar and denote them by $L^{H}$. The parameter $\alpha$ is determined to be the parameter of the density of the driving Lévy process. In the next proposition we give conditions under which such processes are Hölder-continuous. 
Proposition 3. Let $\beta:=\inf \left\{u \geq\left. 0\left|\int_{\mathbb{R}} 1 \wedge\right| x\right|^{u} d v(x)<\infty\right\} \in(0,2)$ be the BlumenthalGetoor index of the driving Lévy process of $L^{H}$, then if $\alpha>1, \beta<\alpha$ and $\gamma \in\left(0,1-\frac{1}{\alpha}\right)$ the process $L^{H}$ is Hölder-continuous of order d with $d<\gamma$.

Proof. See [EW13], Proposition 6.

Before we start considering our main goals, we need to prove the following integral representation for the power-function (cf. [BCI04]).

Lemma 2. Let $x \in \mathbb{R}$. Then for all $p \in(0,2)$ :

$$
|x|^{p}=\frac{\int_{\mathbb{R}}\left(e^{i y x}-1-i y x \mathbb{1}_{|y| \leq 1}(y)\right)|y|^{-(1+p)} d y}{\int_{\mathbb{R}}\left(e^{i y}-1-i y \mathbb{1}_{|y| \leq 1}(y)\right)|y|^{-(1+p)} d y} .
$$

Proof. The result is derived by substituting $z=|x| y$ in the upper integral and observing, that the integral in the numerator does not depend on the sign of $x$.

\section{LAW OF LARGE NUMBERS FOR POWER VARIATION OF LOCAL SELF-SIMILAR FRACTIONAL LÉVY PROCESSES}

In this section we want to prove our main result, namely a law of large numbers for power variations of integrated fractional Lévy processes. The power variation of a process $Z$ is defined by

$$
V_{p}^{n}(Z)_{t}:=\sum_{j=1}^{\lfloor n t\rfloor}\left|\left(Z_{\frac{j}{n}}-Z_{\frac{j-1}{n}}\right)\right|^{p} .
$$

In our case we look at processes of the form

$$
Z_{t}=\int_{0}^{t} u_{s} d L_{s}^{H}
$$

where $u$ is a stochastic process. We first need to make sure that the process $Z$ exists. Let $c_{p, q}:=$ $\zeta\left(\frac{1}{p}+\frac{1}{q}\right)$ with $\zeta$ the Riemann-Zeta function. In [You36] Young proved that the Riemann-Stieltjes integral of $f$ with respect to $g$ exists if the functions $f$ and $g$ have finite $p$-, resp. $q$-variation and $\frac{1}{p}+\frac{1}{q}>1$. This is because of the Young inequality

$$
\left|\int_{a}^{b} f d g-f(a)(g(b)-g(a))\right| \leq c_{p, q} \operatorname{var}_{p}(f ;[a, b]) \operatorname{var}_{q}(g ;[a, b])
$$


where $\operatorname{var}_{p}(f ;[a, b])$ is the $p$-variation of a function $f$ on an interval $[a, b]$, defined as

$$
\operatorname{var}_{p}(f ;[a, b]):=\sup _{\pi}\left(\sum_{i=1}^{n}\left|f\left(t_{i}\right)-f\left(t_{i-1}\right)\right|^{p}\right)^{\frac{1}{p}},
$$

where the supremum is taken over all partitions $\pi=\left\{a \leq t_{0}<\cdots<t_{n} \leq b\right\}$ of the interval $[a, b]$. If a function has $\alpha$-Hölder continuous paths it has finite $\frac{1}{\alpha}$-variation. In Proposition 3 we showed that under $\alpha>1$ and $\beta<\alpha$ fractional Lévy processes are Hölder-continuous of order $\gamma-\varepsilon$ for any $0<\varepsilon<\gamma$, where $\beta$ is the Blumenthal-Getoor index of the driving Lévy process. If these conditions are satisfied the integral of $u$ with respect to $L^{H}$ exists if the process $u$ has finite $q$-variation with $q<\frac{1}{1-\gamma}$. This is where we need to be more restrictive because fractional Brownian motions are Hölder-continuous of order $H$ and its always $H>\gamma$, so the process $u$ needs to be more regular in our case. For the sake of completeness we set

$$
\|f\|_{\gamma-\varepsilon ;[a, b]}:=\sup _{a \leq s<t \leq b} \frac{\left|f_{t}-f_{s}\right|}{|t-s|^{\gamma-\varepsilon}}
$$

With these tools we are now able to state the next theorem. This is a generalization of Theorem 1 of [CNW06] where the result is shown in the Gaussian case.

Theorem 1. Let $L^{H}$ be a local self similar fractional Lévy process and suppose that for the Blumenthal-Getoor index $\beta$ of the driving Lévy process $L$ of $L^{H}$ it holds that $\beta<\alpha$. Let $X$ be an $\alpha$-stable linear fractional stable motion as in Example 1 and let also $u=\left(u_{t}\right)_{t \in[0, T]} \in L^{p}([0, T])$ be a stochastic process with a.s. finite q-variation, where $q<\frac{1}{1-\gamma}$. Consider the process

$$
Z_{t}=\int_{0}^{t} u_{s} d L_{s}^{H}
$$

Then, if $n$ tends to infinity, the following holds:

$$
n^{-1+p H} V_{p}^{n}(Z)_{t} \stackrel{\text { u.c.p }}{\longrightarrow} \mathbb{E}\left[\left|X_{1}\right|^{p}\right] \int_{0}^{t}\left|u_{s}\right|^{p} d s .
$$

Before proving this theorem we consider the case $u \equiv 1$. In contrast to the Gaussian case in [CNW06] this is the most complicated step in our proof. That is why we consider this case separated from our main result. For the sake of simplicity in the next theorem, we define

$$
V_{t}^{n}:=n^{-1+p H} V_{p}^{n}\left(L^{H}\right)_{t}=\frac{1}{n} \sum_{j=1}^{\lfloor n t\rfloor}\left|n^{H}\left(L_{\frac{j}{n}}^{H}-L_{\frac{j-1}{n}}^{H}\right)\right|^{p} .
$$

Theorem 2. The power-variation $V_{t}^{n}$ of a local self similar fractional Lévy process $L^{H}$ converges for all $p<\alpha$ as $n \rightarrow \infty$

$$
V_{t}^{n} \stackrel{\mathbb{P}}{\rightarrow} t \cdot \mathbb{E}\left[\left|X_{1}\right|^{p}\right],
$$

where the process $X$ is the linear fractional stable motion as in example 1. 
Proof. For the proof we will proceed in two steps. At first we show, that the expectation of $V_{t}^{n}$ converges to $t \mathbb{E}\left[\left|X_{1}\right|^{p}\right]$. After that we proof a law of large numbers for the power variation of fractional Lévy-process $L^{H}$ to show that $V_{t}^{n}$ converges in probability to its expectation.

We first consider the expectation of $V_{t}^{n}$. Let therefore $p<\alpha$ and $q>1$ such that $p q<\alpha$. Then the random variables $\left|n^{H}\left(L_{\frac{i}{n}}^{H}-L_{\frac{i-1}{n}}^{H}\right)\right|^{p q}$ are integrable and since the process $L^{H}$ has stationary increments for each $n$ we obtain

$$
\mathbb{E}\left[\left|n^{H}\left(L_{\frac{i}{n}}^{H}-L_{\frac{i-1}{n}}^{H}\right)\right|^{p q}\right]=\mathbb{E}\left[\left|n^{H} L_{\frac{1}{n}}^{H}\right|^{p q}\right]<\infty .
$$

This together with the fact

$$
\mathbb{E}\left(\left|n^{H}\left(L_{\frac{i}{n}}^{H}-L_{\frac{i-1}{n}}^{H}\right)\right|^{p} ;\left|n^{H}\left(L_{\frac{i}{n}}^{H}-L_{\frac{i-1}{n}}^{H}\right)\right|^{p}>r\right) \leq r^{-q+1} \mathbb{E}\left(\left|n^{H}\left(L_{\frac{i}{n}}^{H}-L_{\frac{i-1}{n}}^{H}\right)\right|^{p q}\right)
$$

yields the uniform integrability of the sequence and by Lemma 4.11 of [Kal10] the following convergence holds:

$$
\mathbb{E}\left(\left|n^{H}\left(L_{\frac{i}{n}}^{H}-L_{\frac{i-1}{n}}^{H}\right)\right|^{p}\right) \stackrel{n \rightarrow \infty}{\rightarrow} \mathbb{E}\left(\left|X_{1}\right|^{p}\right) .
$$

Then with Proposition 2 we can conclude

$$
\begin{aligned}
\mathbb{E}\left[V_{t}^{n}\right] & =\frac{1}{n} \sum_{j=1}^{\lfloor n t\rfloor} \mathbb{E}\left[\left|n^{H}\left(L_{\frac{j}{n}}^{H}-L_{\frac{j-1}{n}}^{H}\right)\right|^{p}\right] \\
& =\frac{\lfloor n t\rfloor}{n} \mathbb{E}\left[\left|n^{H} L_{\frac{1}{n}}^{H}\right|^{p}\right] \\
& \rightarrow \substack{\rightarrow \rightarrow \infty} \mathbb{E}\left[\left|X_{1}\right|^{p}\right] .
\end{aligned}
$$

Now we start with the second step. For shorter notations, we denote by $N$ the numerator in the integral representation of Lemma 2, this is

$$
N:=\int_{\mathbb{R}}\left(e^{i y}-1-i y \mathbb{1}_{|y| \leq 1}(y)\right)|y|^{-(1+p)} d y .
$$

We now use Lemma 2 and conclude

(2)

$$
V_{t}^{n}=\frac{1}{N} \int_{\mathbb{R}}\left(\frac{1}{n} \sum_{j=1}^{\lfloor n t\rfloor} e^{i y n^{H}\left(L_{\frac{j}{n}}^{H}-L_{\frac{j-1}{n}}^{H}\right)}-\frac{\lfloor n t\rfloor}{n}-i y n^{H} \frac{1}{n} \sum_{j=1}^{\lfloor n t\rfloor}\left(L_{\frac{j}{n}}^{H}-L_{\frac{j-1}{n}}^{H}\right) \mathbb{1}_{|y| \leq 1}\right) \frac{d y}{|y|^{p+1}} .
$$

We first have to consider the two cases $p<1$ and $p \geq 1$. In the first case the integral $\int_{-1}^{1} \frac{i y x}{|y|^{p+1}} d y$ exists for any $x$ and its value is 0 because of the symmetry of the integrand, hence we have in the 
case $p<1$

$$
V_{t}^{n}=\frac{1}{N} \int_{\mathbb{R}}\left(\frac{1}{n} \sum_{j=1}^{\lfloor n t\rfloor} e^{i y n^{H}\left(L_{\frac{j}{n}}^{H}-L_{\frac{j-1}{n}}^{H}\right)}-\frac{\lfloor n t\rfloor}{n}\right) \frac{d y}{|y|^{p+1}}
$$

and

$$
\mathbb{E}\left[V_{t}^{n}\right]=\mathbb{E}\left[\frac{1}{N} \int_{\mathbb{R}}\left(\frac{1}{n} \sum_{j=1}^{\lfloor n t\rfloor} e^{i y n^{H}\left(L_{\frac{j}{n}}^{H}-L_{\frac{j-1}{n}}^{H}\right)}-\frac{\lfloor n t\rfloor}{n}\right) \frac{d y}{|y|^{p+1}}\right],
$$

this yields

$$
V_{t}^{n}-\mathbb{E}\left[V_{t}^{n}\right]=\frac{1}{N} \int_{\mathbb{R}} \frac{1}{n} \sum_{j=1}^{\lfloor n t\rfloor}\left(e^{i y n^{H}\left(L_{\frac{j}{n}}^{H}-L_{\frac{j-1}{n}}^{H}\right)}-\mathbb{E}\left[e^{i y n^{H}\left(L_{\frac{j}{n}}^{H}-L_{\frac{j-1}{n}}^{H}\right)}\right]\right) \frac{d y}{|y|^{p+1}} .
$$

In the case $p \geq 1$ the process $L^{H}$ has finite first moments and we can use Fubini's theorem. This yields

$$
\begin{aligned}
V_{t}^{n}-\mathbb{E}\left[V_{t}^{n}\right] & \frac{1}{N} \int_{\mathbb{R}}\left(\frac { 1 } { n } \sum _ { j = 1 } ^ { \lfloor n t \rfloor } \left(e^{i y n^{H}\left(L_{\frac{j}{n}}^{H}-L_{\frac{j-1}{n}}^{H}\right)}-\mathbb{E}\left[e^{\left.\left.i y n^{H}\left(L_{\frac{j}{n}}^{H}-L_{\frac{j-1}{n}}^{H}\right)\right]\right)}\right.\right.\right. \\
& \left.-i y n^{H} \frac{1}{n} \sum_{j=1}^{\lfloor n t\rfloor}\left(\left(L_{\frac{j}{n}}^{H}-L_{\frac{j-1}{n}}^{H}\right)-\mathbb{E}\left[L_{\frac{j}{n}}^{H}-L_{\frac{j-1}{n}}^{H}\right]\right) \mathbb{1}_{|y| \leq 1}\right) \frac{d y}{|y|^{p+1}} .
\end{aligned}
$$

The last term is a telescopic sum and since $L_{0}=0$ a.s. it is

$$
i y n^{H} \frac{1}{n} \sum_{j=1}^{\lfloor n t\rfloor}\left(\left(L_{\frac{j}{n}}^{H}-L_{\frac{j-1}{n}}^{H}\right)-\mathbb{E}\left[L_{\frac{j}{n}}^{H}-L_{\frac{j-1}{n}}^{H}\right]\right)=i y \frac{n^{H}}{n}\left(L_{\frac{\lfloor n t\rfloor}{n}}^{H}-\mathbb{E}\left[L_{\frac{\lfloor n t\rfloor}{n}}^{H}\right]\right)
$$

and since $H<1$ this converges to 0 almost surely as $n \rightarrow \infty$. So in the case $p \geq 1$ the difference $V_{t}^{n}-\mathbb{E}\left[V_{t}^{n}\right]$ becomes the same as in the case $p<1$ and is given by equation (3).

Unfortunately we are not able to show

$$
\frac{1}{n} \sum_{j=1}^{\lfloor n t\rfloor} e^{i y n^{H}\left(L_{\frac{j}{n}}^{H}-L_{\frac{j-1}{n}}^{H}\right)}-\mathbb{E}\left[e^{i y n^{H}\left(L_{\frac{j}{n}}^{H}-L_{\frac{j-1}{n}}^{H}\right)}\right] \rightarrow 0 \quad \mathbb{P}-\text { a.s. }
$$

as $n \rightarrow \infty$. If we could do this, we would be able to use Lebesgue's theorem to show the almost sure convergence of $V_{t}^{n}$ to its expectation. But if we can show convergence in probability in equation (4) we can conclude $V_{t}^{n} \stackrel{\mathbb{P}}{\rightarrow} \mathbb{E}\left[V_{t}^{n}\right]$. This holds by the fact that convergence in probability of a sequence $\left(\xi_{n}\right)_{n \in \mathbb{N}}$ of random variables to a random variable $\xi$ is equivalent to the fact that for all subsequences $\left(\xi_{n_{k}}\right)_{k \in \mathbb{N}}$ there exists a subsubsequence $\left(\xi_{n_{k_{l}}}\right)_{l \in \mathbb{N}}$ such that $\xi_{n_{k_{l}}} \rightarrow \xi \mathbb{P}$-a.s. for 
$l \rightarrow \infty$. If we take an arbitrary subsequence $V_{t}^{n_{k}}-\mathbb{E}\left[V_{t}^{n_{k}}\right]$ of $V_{t}^{n}-\mathbb{E}\left[V_{t}^{n}\right]$ we can take an almost sure convergent subsubsequence of the resulting term of the left side of equation (4), namely

$$
\frac{1}{n_{k_{l}}} \sum_{j=1}^{\left\lfloor n_{k_{l}}\right\rfloor} e^{i y n_{k_{l}}^{H}\left(L_{\frac{j}{n_{k_{l}}}}^{H}-L_{\frac{j-1}{n_{k_{l}}}}^{H}\right)}-\mathbb{E}\left[e^{i y n_{k_{l}}^{H}\left(L_{\frac{j}{n_{k_{l}}}}^{H}-L_{\frac{j-1}{n_{k_{l}}}}^{H}\right)}\right]
$$

and we can apply Lebesgue's theorem for this subsubsequence. This yields the convergence in probability of $V_{t}^{n}$ to its expectation.

Hence it remains to show convergence in probability in equation (4). To show this we prove $L^{2}$-convergence. We use the stationarity of the fractional Lévy process and conclude

$$
\begin{aligned}
& \mathbb{E}\left[\mid \frac{1}{n} \sum_{j=1}^{\lfloor n t\rfloor}\left(e^{i y n^{H}\left(L_{\frac{j}{n}}^{H}-L_{\frac{j-1}{n}}^{H}\right)}-\mathbb{E}\left[e^{\left.i y n^{H}\left(L_{\frac{j}{n}}^{H}-L_{\frac{j-1}{n}}^{H}\right)\right]}\right)^{2}\right]\right. \\
= & \frac{1}{n^{2}} \sum_{j=1}^{\lfloor n t\rfloor} \sum_{k=1}^{\lfloor n t\rfloor} \operatorname{Cov}\left(e^{i y n^{H}\left(L_{\frac{j}{n}}^{H}-L_{\frac{j-1}{n}}^{H}\right)}, e^{i y n^{H}\left(L_{\frac{k}{n}}^{H}-L_{\frac{k-1}{n}}^{H}\right)}\right) \\
\leq & \frac{2}{n^{2}} \sum_{j=1}^{\lfloor n t\rfloor} \sum_{k=1}^{j} \operatorname{Cov}\left(e^{i y n^{H}\left(\frac{L^{H}}{\frac{j-k+1}{n}}-L_{\frac{j-k}{n}}^{H}\right)}, e^{i y n^{H} L_{\frac{1}{n}}^{H}}\right) .
\end{aligned}
$$

For further notations, we define $a:=j-k$. If we show that the last expression is $\mathcal{O}\left(a^{-\delta}\right)$ for some $\delta>0$ this yields the $L^{2}$-convergence in equation (4). To this end we first use the characteristic functions of the process $L^{H}$ (cf. Proposition 1) and use similar substitutions as in the proof of Proposition 2 to calculate 


$$
\begin{aligned}
& \operatorname{Cov}\left(e^{i y n^{H}\left(L_{\frac{a+1}{n}}^{H}-L_{\frac{a}{n}}^{H}\right)}, e^{i y n^{H} L_{\frac{1}{n}}^{H}}\right) \\
& =\mathbb{E}\left[e^{i y n^{H}\left(L_{\frac{a+1}{n}}^{H}-L_{\frac{a}{n}}^{H}+L_{\frac{1}{n}}^{H}\right)}\right]-\mathbb{E}\left[e^{i y n^{H}\left(L_{\frac{a+1}{n}}^{H}-L_{\frac{a}{n}}^{H}\right)}\right] \mathbb{E}\left[e^{i y n^{H} L_{\frac{1}{n}}^{H}}\right] \\
& =\exp \left\{\int _ { \mathbb { R } ^ { 2 } } \left(e^{i y x n^{H}\left(\left(\frac{a+1}{n}-s\right)_{+}^{\gamma}-\left(\frac{a}{n}-s\right)_{+}^{\gamma}+\left(\frac{1}{n}-s\right)_{+}^{\gamma}-(-s)_{+}^{\gamma}\right)-1}\right.\right. \\
& \left.\left.-i y x n^{H}\left(\left(\frac{a+1}{n}-s\right)_{+}^{\gamma}-\left(\frac{a}{n}-s\right)_{+}^{\gamma}+\left(\frac{1}{n}-s\right)_{+}^{\gamma}-(-s)_{+}^{\gamma}\right) \mathbb{1}_{|x| \leq 1}\right) d v(x) d s\right\} \\
& -\exp \left\{\int _ { \mathbb { R } ^ { 2 } } \left(e^{i y x n^{H}\left(\left(\frac{a+1}{n}-s\right)_{+}^{\gamma}-\left(\frac{a}{n}-s\right)_{+}^{\gamma}\right)}+e^{\left(\left(\frac{1}{n}-s\right)_{+}^{\gamma}-(-s)_{+}^{\gamma}\right)}-2\right.\right. \\
& \left.\left.-i y x n^{H}\left(\left(\frac{a+1}{n}-s\right)_{+}^{\gamma}-\left(\frac{a}{n}-s\right)_{+}^{\gamma}+\left(\frac{1}{n}-s\right)_{+}^{\gamma}-(-s)_{+}^{\gamma}\right) \mathbb{1}_{|x| \leq 1}\right) d v(x) d s\right\}
\end{aligned}
$$

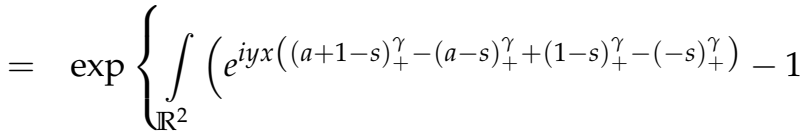

$$
\begin{aligned}
& \left.\left.-i y x\left((a+1-s)_{+}^{\gamma}-(a-s)_{+}^{\gamma}+(1-s)_{+}^{\gamma}-(-s)_{+}^{\gamma}\right) \mathbb{1}_{|x| \leq 1}\right) \frac{1}{n} d v\left(x n^{-1 / \alpha}\right) d s\right\} \\
& -\exp \left\{\int _ { \mathbb { R } ^ { 2 } } \left(e^{i y x\left((a+1-s)_{+}^{\gamma}-(a-s)_{+}^{\gamma}\right)}+e^{\left((1-s)_{+}^{\gamma}-(-s)_{+}^{\gamma}\right)}-2\right.\right. \\
& \left.\left.-i y x\left((a+1-s)_{+}^{\gamma}-(a-s)_{+}^{\gamma}+(1-s)_{+}^{\gamma}-(-s)_{+}^{\gamma}\right) \mathbb{1}_{|x| \leq 1}\right) \frac{1}{n} d v\left(x n^{-1 / \alpha}\right) d s\right\},
\end{aligned}
$$

where $v$ is the Lévy-measure of the driving Lévy process $L$ of $L^{H}$, which is absolutely continuous with respect to Lebesgue-measure with density $g$. This density $g$ has the properties given in Proposition 2.

For shorter notations we also define $z_{a}(s):=(a+1-s)_{+}^{\gamma}-(a-s)_{+}^{\gamma}$. To go on with the proof we use the continuity of the exponential-function so we can consider the exponents of the last expression. Also we use the second property of the density $g$, this is $g(x) \leq C \frac{1}{|x|^{1+\alpha}}$. 


$$
\begin{aligned}
& \int_{\mathbb{R}^{2}}\left(e^{i y x\left(z_{a}(s)+z_{0}(s)\right)}-1-i y x\left(z_{a}(s)+z_{0}(s)\right) \mathbb{1}_{|x| \leq 1}\right) \frac{1}{n} d v\left(x n^{-1 / \alpha}\right) d s \\
- & \int_{\mathbb{R}^{2}}\left(e^{i y x z_{a}(s)}+e^{i y x z_{0}(s)}-2-i y x\left(z_{a}(s)+z_{0}(s)\right) \mathbb{1}_{|x| \leq 1}\right) \frac{1}{n} d v\left(x n^{-1 / \alpha}\right) d s \mid \\
\leq & \int_{\mathbb{R}^{2}}\left|e^{i y x\left(z_{a}(s)+z_{0}(s)\right)}-e^{i y x z_{a}(s)}-e^{i y x z_{0}(s)}+1\right| \frac{1}{n} d v\left(x n^{-1 / \alpha}\right) d s \\
\leq & C \int_{\mathbb{R}^{2}}\left|e^{i y x\left(z_{a}(s)+z_{0}(s)\right)}-e^{i y x z_{a}(s)}-e^{i y x z_{0}(s)}+1\right| \frac{1}{|x|^{1+\alpha}} d x d s \\
= & \int_{\mathbb{R}^{2}} \mid \cos \left(y x\left(z_{a}(s)+z_{0}(s)\right)\right)-\cos \left(y x z_{a}(s)\right)-\cos \left(y x z_{0}(s)\right)+1 \\
& +i\left[\sin \left(y x\left(z_{a}(s)+z_{0}(s)\right)\right)-\sin \left(y x z_{a}(s)\right)-\sin \left(y x z_{0}(s)\right)\right] \mid \frac{1}{|x|^{1+\alpha}} d x d s
\end{aligned}
$$

We can clearly see, that the integrands are the same if $s>1$, so the expression is 0 for $s>1$. Using the standard addition theorems for sine and cosine functions we get

$$
\begin{aligned}
& \int_{\mathbb{R}^{2}} \mid \cos y x\left(z_{a}(s)+z_{0}(s)\right)-\cos y x z_{a}(s)-\cos y x z_{0}(s)+1 \\
& \quad+i\left[\sin y x\left(z_{a}(s)+z_{0}(s)\right)-\sin y x z_{a}(s)-\sin y x z_{0}(s)\right] \mid \frac{1}{|x|^{1+\alpha}} d x d s \\
& =\int_{-\infty}^{1} \int_{\mathbb{R}} \mid 1-\cos y x z_{a}(s)+\cos y x z_{0}(s)\left(\cos y x z_{a}(s)-1\right)-\sin y x z_{a}(s) \sin y x z_{0}(s) \\
& \quad+i\left[\sin y x z_{a}(s)\left(\cos y x z_{0}(s)-1\right)-\sin y x z_{0}(s)\left(\cos y x z_{a}(s)-1\right)\right] \mid \frac{1}{|x|^{1+\alpha}} d x d s \\
& \leq \int_{-\infty}^{1} \int_{\mathbb{R}}\left(3\left|\cos y x z_{a}(s)-1\right|+\left|\sin y x z_{a}(s)\right|\left|\sin y x z_{0}(s)\right|\right. \\
& \left.\quad+\left|\sin y x z_{a}(s)\right|\left|1-\cos y x z_{0}(s)\right|\right) \frac{1}{|x|^{1+\alpha}} d x d s
\end{aligned}
$$

Now we use in all of the cases the estimations $|\sin x| \leq 1 \wedge|x|$ and $|\cos x-1| \leq 2 \wedge \frac{|x|^{2}}{2}$ for sine and cosine functions and we decompose the integrals as follows: 
For the first summand we obtain

$$
\begin{aligned}
& \int_{\mathbb{R}}\left|\cos y x z_{a}(s)-1\right| \frac{d x}{|x|^{1+\alpha}} \\
\leq & \int_{\mathbb{R}}\left(\frac{\left|y x z_{a}(s)\right|^{2}}{2} \wedge 2\right) \frac{d x}{|x|^{1+\alpha}} \\
= & 2 \frac{\left|y z_{a}(s)\right|^{2}}{2} \int_{0}^{\frac{2}{\left|y z_{a}(s)\right|}} x^{1-\alpha} d x+2 \int_{\frac{2}{\left|y z_{a}(s)\right|}}^{\infty} 2 x^{-1-\alpha} d x \\
= & \frac{\left|y z_{a}(s)\right|^{2}}{2-\alpha}\left(\frac{2}{\left|y z_{a}(s)\right|}\right)^{2-\alpha}+\frac{4}{\alpha}\left(\frac{2}{\left|y z_{a}(s)\right|}\right)^{-\alpha} \\
= & \operatorname{const}\left|y z_{a}(s)\right|^{\alpha}
\end{aligned}
$$

For the second term we use similar techniques and conclude

$$
\begin{aligned}
& \int_{\mathbb{R}}\left|\sin y x z_{a}(s)\right|\left|\sin y x z_{0}(s)\right| \frac{d x}{|x|^{1+\alpha}} \\
\leq & \int_{\mathbb{R}}\left(\left|y x z_{a}(s)\right| \wedge 1\right)\left(\left|y x z_{0}(s)\right| \wedge 1\right) \frac{d x}{|x|^{1+\alpha}} \\
= & 2 \int_{0}^{\left|y z_{0}(s)\right|^{-1}}\left|y x z_{a}(s)\right|\left|y x z_{0}(s)\right| \frac{d x}{|x|^{1+\alpha}}+2 \int_{\left|y z_{0}(s)\right|^{-1}}^{\left|y x z_{a}(s)\right|^{-1}}(s) \mid \frac{d x}{|x|^{1+\alpha}}+2 \int_{\left|y z_{a}(s)\right|^{-1}}^{\infty} \frac{d x}{|x|^{1+\alpha}} \\
= & \frac{2|y|^{2}\left|z_{a}(s) z_{0}(s)\right|}{2-\alpha}\left|y z_{0}(s)\right|^{-2+\alpha}+\frac{2\left|y z_{a}(s)\right|}{1-\alpha}\left(\left|y z_{a}(s)\right|^{\alpha-1}-\left|y z_{0}(s)\right|^{\alpha-1}\right)+\frac{2}{\alpha}\left|y z_{a}(s)\right|^{\alpha} \\
= & \operatorname{const}\left|z_{a}(s)\right||y|^{\alpha}\left|z_{0}(s)\right|^{\alpha-1}+\operatorname{const}^{\alpha}\left|y z_{a}(s)\right|^{\alpha} .
\end{aligned}
$$

The last term is easy to handle, because

$$
\left|1-\cos y x z_{0}(s)\right| \leq \frac{\left|y x z_{0}(s)\right|^{2}}{2} \wedge 2=2\left(\left(\frac{\left|y x z_{0}(s)\right|}{2}\right)^{2} \wedge 1\right) \leq 2\left(\left|y x z_{0}(s)\right| \wedge 1\right),
$$

so in fact this is smaller than a constant times the estimation of the second integrand.

Finally the last step is to show, that both $\int_{-\infty}^{1}\left|y z_{a}(s)\right|^{\alpha} d s$ and $\int_{-\infty}^{1} \frac{\left|z_{a}(s)\right|}{\left|z_{0}(s)\right|}\left|y z_{0}(s)\right|^{\alpha} d s$ are $\mathcal{O}\left(a^{-\delta}\right)$ for $a \rightarrow \infty$ and that both integrals are finite. The first observation is that $z_{a}(s)$ is monotone increasing in $s$ on the interval $(-\infty, a]$ and because of the behaviour on the interval $[a, a+1]$, it is $\frac{\left|z_{a}(s)\right|}{\left|z_{0}(s)\right|} \leq 1$ for all $s \in(-\infty, 1], \gamma \in\left(-\frac{1}{\alpha}, 1-\frac{1}{\alpha}\right)$ and $a \geq 2$, and since Lemma 1 holds for those $\gamma$ both integrals are finite. Let $\delta>0$ such that $\gamma+2 \delta<1-\frac{1}{\alpha}$. If we replace $\gamma$ by $\gamma+2 \delta$, the 
integrals are still finite and we calculate for the first term and for all $s \leq 1$

$$
\begin{aligned}
z_{a}(s) & =(a+1-s)_{+}^{-2 \delta}(a+1-s)_{+}^{\gamma+2 \delta}-(a-s)_{+}^{-2 \delta}(a-s)_{+}^{\gamma+2 \delta} \\
& \leq(a-1)^{-2 \delta}\left((a+1-s)^{\gamma+2 \delta}-(a-s)^{\gamma+2 \delta}\right) .
\end{aligned}
$$

On the other hand

$$
z_{0}(s) \geq(1-s)^{-2 \delta}\left((1-s)^{\gamma+2 \delta}-(-s)_{+}^{\gamma+2 \delta}\right)
$$

For $s \in(-\infty, 1]$, both of these inequalities yield

$$
\begin{aligned}
\frac{\left|z_{a}(s)\right|}{\left|z_{0}(s)\right|} & =\frac{(a+1-s)_{+}^{-2 \delta}(a+1-s)_{+}^{\gamma+2 \delta}-(a-s)_{+}^{-2 \delta}(a-s)_{+}^{\gamma+2 \delta}}{(1-s)_{+}^{-2 \delta}(1-s)_{+}^{\gamma+2 \delta}-(-s)_{+}^{-2 \delta}(-s)_{+}^{\gamma+2 \delta}} \\
& \leq \frac{(a-s)^{-2 \delta}}{(1-s)^{-2 \delta}} \cdot \frac{(a+1-s)^{\gamma+2 \delta}-(a-s)^{\gamma+2 \delta}}{(1-s)^{\gamma+2 \delta}-(-s)_{+}^{\gamma+2 \delta}} \\
& \leq\left(1+\frac{a-1}{1-s}\right)^{-2 \delta} .
\end{aligned}
$$

Unfortunately it is not $\frac{\left|z_{a}(s)\right|}{\left|z_{0}(s)\right|}=\mathcal{O}\left(a^{-\delta}\right)$, so we need to split the integral into two parts, the part on the interval $(-\infty,-R]$ and the interval $[-R, 1]$ for some $R>0$ determined later. On the second interval we use estimation (6) of the factor $\frac{\left|z_{a}(s)\right|}{\left|z_{0}(s)\right|}$. On the interval $(-\infty,-R]$ we use $\frac{\left|z_{a}(s)\right|}{\left|z_{0}(s)\right|} \leq 1$ and the mean value theorem: For all $s \in(-\infty, 0]$ there exists $\xi \in[0,1]$, such that $(1-s)_{+}^{\gamma}-(-s)_{+}^{\gamma}=\gamma(\xi-s)^{\gamma-1}$. Since $\gamma-1<0$ we can estimate

$$
(1-s)_{+}^{\gamma}-(-s)_{+}^{\gamma} \leq \gamma(1-s)^{\gamma-1}
$$

Both of this yields

$$
\begin{aligned}
\int_{-\infty}^{1}\left|z_{0}(s)\right|^{\alpha}\left|\frac{z_{a}(s)}{z_{0}(s)}\right| d s & =\int_{-\infty}^{-R}\left|z_{0}(s)\right|^{\alpha}\left|\frac{z_{a}(s)}{z_{0}(s)}\right| d s+\int_{-R}^{1}\left|z_{0}(s)\right|^{\alpha}\left|\frac{z_{a}(s)}{z_{0}(s)}\right| d s \\
& \leq\left|\frac{\gamma}{\gamma \alpha-\alpha+1}\right|(1+R)^{\gamma \alpha-\alpha+1}+\left(1+\frac{a-1}{1+R}\right)^{-2 \delta} \int_{-R}^{1}\left|z_{0}(s)\right|^{\alpha} d s .
\end{aligned}
$$

We now observe that $\gamma \alpha-\alpha+1<-2 \delta$ so if we choose $R>0$ such that $1+R=(a-1)^{\frac{1}{2}}$ we can estimate

$$
c_{1}(1+R)^{\gamma \alpha-\alpha+1}+c_{2}\left(1+\frac{a-1}{1+R}\right)^{-2 \delta} \leq c_{1}(a-1)^{-\delta}+c_{2}\left(1+(a-1)^{\frac{1}{2}}\right)^{-2 \delta}=\mathcal{O}\left(a^{-\delta}\right) .
$$

For the first integral things are easier. Equation (5) yields 
$\int_{-\infty}^{1}\left|y z_{a}(s)\right|^{\alpha} d s \leq|y|^{\alpha}(a-1)^{-2 \delta} \int_{-\infty}^{1}(a+1-s)^{\gamma+2 \delta}-(a-s)^{\gamma+2 \delta} d s=\mathcal{O}\left(a^{-2 \delta}\right)=\mathcal{O}\left(a^{-\delta}\right)$.

Hence we can conclude

$$
\operatorname{Cov}\left(e^{i y\left(X_{a+1}-X_{a}\right)}, e^{i y\left(X_{1}-X_{0}\right)}\right)=\mathcal{O}\left(a^{-\delta}\right)=\mathcal{O}\left((j-k)^{-\delta}\right) \quad \text { for } a \rightarrow \infty
$$

and hence $V_{t}^{n}$ converges to $t \mathbb{E}\left[\left|X_{1}\right|^{p}\right]$ in $L^{2}$ and so it converges in probability.

Now we can start with the proof of Theorem 1:

Proof of Theorem 1. Fix $T \in \mathbb{R}$ and let $t \in[0, T]$. We define $c_{p}:=\mathbb{E}\left[\left|X_{1}\right|^{p}\right]$ and consider at first the case $p \leq 1$ and conclude for all $m \geq n$,

$$
\begin{aligned}
& m^{-1+p H} V_{p}^{m}(Z)_{t}-c_{p} \int_{0}^{t}\left|u_{s}\right|^{p} d s \\
= & \left.m^{-1+p H} \sum_{j=1}^{\lfloor m t\rfloor}\left|\int_{(j-1) / m}^{j / m} u_{s} d L_{s}^{H}\right|^{p}-\left|u_{\frac{j-1}{m}}\left(L_{\frac{j}{m}}^{H}-L_{\frac{j-1}{m}}^{H}\right)\right|^{p}\right) \\
& +m^{-1+p H}\left(\sum_{j=1}^{\lfloor m t\rfloor}\left|u_{\frac{j-1}{m}}\left(L_{\frac{j}{m}}^{H}-L_{\frac{j-1}{m}}^{H}\right)\right|^{p}-\sum_{i=1}^{\lfloor n t\rfloor}\left|u_{\frac{i-1}{n}}\right|^{p} \sum_{j \in I_{n}(i)}\left|L_{\frac{j}{m}}^{H}-L_{\frac{j-1}{m}}^{H}\right|^{p}\right) \\
& +m^{-1+p H} \sum_{i=1}^{\lfloor n t\rfloor}\left|u_{\frac{i-1}{n}}\right|^{p} \sum_{j \in I_{n}(i)}\left|L_{\frac{j}{m}}^{H}-L_{\frac{j-1}{m}}^{H}\right|^{p}-c_{p} n^{-1} \sum_{i=1}^{\frac{\lfloor n t}{m}\left|u_{\frac{i-1}{n}}\right|^{p}} \\
& +c_{p}\left(n^{-1} \sum_{i=1}^{\lfloor n t\rfloor}\left|u_{\frac{i-1}{n}}\right|^{p}-\int_{0}^{t}\left|u_{s}\right|^{p} d s\right) \\
=: & A_{t}^{(m)}+B_{t}^{(n, m)}+C_{t}^{(n, m)}+D_{t}^{(n)},
\end{aligned}
$$

where

$$
I_{n}(i)=\left\{j \in \mathbb{N} \mid \frac{j}{m} \in\left(\frac{i-1}{n}, \frac{i}{n}\right]\right\}, \quad 1 \leq i \leq\lfloor n t\rfloor .
$$

For any fixed $n \in \mathbb{N}$, the summand $C_{t}^{(n, m)}$ converges to 0 in probability as $m \rightarrow \infty$ by observing

$$
\left\|C_{t}^{(n, m)}\right\|_{\infty} \leq \sum_{i=1}^{\lfloor n t\rfloor}\left|u_{\frac{i-1}{n}}\right|^{p}\left|m^{-1+p H} \sum_{j \in I_{n}(i)}\right| L_{\frac{j}{m}}^{H}-\left.L_{\frac{j-1}{m}}^{H}\right|^{p}-c_{p} n^{-1} \mid
$$


and applying Theorem 2. For the term $B_{t}^{(n, m)}$ we get

$$
\begin{aligned}
\left\|B^{(n, m)}\right\|_{\infty} \leq & \left.m^{-1+p H} \sum_{i=1}^{\lfloor n t\rfloor} \sum_{j \in I_{n}(i)}|| u_{\frac{i-1}{n}}\right|^{p}-\left|u_{\frac{j-1}{m}}\right|^{p}|| L_{\frac{j}{m}}^{H}-\left.L_{\frac{j-1}{m}}^{H}\right|^{p} \\
& +\left\||u|^{p}\right\|_{\infty} \sup _{0 \leq t \leq T} m^{-1+p H} \sum_{m n^{-1}\lfloor n t\rfloor \leq j \leq m n^{-1}(\lfloor n t\rfloor+1)}\left|L_{\frac{j}{m}}^{H}-L_{\frac{j-1}{m}}^{H}\right|^{p} \\
\leq & \left.m^{-1+p H} \sum_{i=1}^{\lfloor n t\rfloor} \sup _{s \in \mathcal{I}_{n}(i) \cup \mathcal{I}_{n}(i-1)}|| u_{\frac{i-1}{n}}\right|^{p}-\left|u_{S}\right|^{p}\left|\sum_{j \in I_{n}(i)}\right| L_{\frac{j}{m}}^{H}-\left.L_{\frac{j-1}{m}}^{H}\right|^{p} \\
& +\left\||u|^{p}\right\|_{\infty} \sup _{0 \leq t \leq T} m^{-1+p H} \sum_{m n^{-1}\lfloor n t\rfloor \leq j \leq m n^{-1}(\lfloor n t\rfloor+1)}\left|L_{\frac{j}{m}}^{H}-L_{\frac{j-1}{m}}^{H}\right|^{p},
\end{aligned}
$$

where we denote

$$
\mathcal{I}_{n}(i):=\left(\frac{i-1}{n}, \frac{i}{n}\right], \quad 1 \leq i \leq\lfloor n t\rfloor
$$

By applying Theorem 2 again we can conclude, that this expression converges in probability to

$$
E_{n}=\frac{c_{p}}{n}\left(\left.\sum_{i=1}^{\lfloor n t\rfloor} \sup _{s \in \mathcal{I}_{n}(i) \cup \mathcal{I}_{n}(i-1)}|| u_{\frac{i-1}{n}}\right|^{p}-\left|u_{s}\right|^{p}\left|+\left\||u|^{p}\right\|_{\infty}\right)\right.
$$

With exactly the same arguments as in [CNW06], this term converges to 0 almost surely. Also the convergence of $\left\|D^{(n)}\right\|_{\infty} \rightarrow 0$ as $n \rightarrow \infty$ is already shown in [CNW06]. For the last part, namely $A_{t}^{(n)}$, we can use the Young inequality and obtain for any $p \leq 1$

$$
\begin{aligned}
\left|A_{t}^{(m)}\right| & \leq m^{-1+p H}\left|\sum_{j=1}^{\lfloor m t\rfloor}\left(\left|\int_{\frac{j-1}{m}}^{\frac{j}{m}} u_{s} d L_{s}^{H}\right|^{p}-\left|u_{\frac{j-1}{m}}\left(L_{\frac{j}{m}}^{H}-L_{\frac{j-1}{m}}^{H}\right)\right|^{p}\right)\right| \\
& \leq m^{-1+p H} \sum_{j=1}^{\lfloor m t\rfloor} \mid \int_{\frac{j-1}{m}}^{\frac{j}{m}} u_{s} d L_{s}^{H}-u_{\frac{j-1}{m}}\left(L_{\frac{j}{m}}^{H}-\left.L_{\frac{j-1}{m}}^{H}\right|^{p}\right. \\
& \leq c_{p^{*}, q} m^{-1+p H} \sum_{j=1}^{\lfloor m t\rfloor}\left(\operatorname{var}_{q}\left(u ; \mathcal{I}_{m}(j)\right) \operatorname{var}_{\frac{1}{\gamma-\varepsilon}}\left(L^{H} ; \mathcal{I}_{m}(j)\right)\right)^{p} \\
& =: \quad c_{p^{*}, q} F_{m},
\end{aligned}
$$


where $p^{*}:=\gamma-\varepsilon$ for $0<\varepsilon<\gamma$. For $\delta>0$ we now consider the decomposition

$$
\begin{aligned}
F_{m} \leq & m^{-1+p H} \sum_{j: \operatorname{var}_{q}\left(u ; \mathcal{I}_{m}(j)\right)>\delta}\left(\operatorname{var}_{q}\left(u ; \mathcal{I}_{m}(j)\right) \operatorname{var}_{\frac{1}{\gamma-\varepsilon}}\left(L^{H} ; \mathcal{I}_{m}(j)\right)\right)^{p} \\
& +\delta^{p} m^{-1+p H} \sum_{j=1}^{\lfloor m t\rfloor}\left(\operatorname{var}_{\frac{1}{\gamma-\varepsilon}}\left(L^{H} ; \mathcal{I}_{m}(j)\right)\right)^{p} .
\end{aligned}
$$

Since

$$
\sum_{j=1}^{\lfloor m t\rfloor} \operatorname{var}_{q}\left(u ; \mathcal{I}_{m}(j)\right) \leq \operatorname{var}_{q}(u ;[0, T])<\infty
$$

we can conclude that the number of indices $j$ for which $\operatorname{var}_{q}\left(u ; \mathcal{I}_{m}(j)\right)>\delta$ holds is bounded by $\left\lfloor\frac{\operatorname{var}_{q}(u ;[0, T])}{\delta}\right\rfloor+1=: M$ and hence

$$
\begin{aligned}
F_{m} \leq & M m^{-1+p H} \max _{1 \leq j \leq\lfloor m T\rfloor}\left(\operatorname{var}_{q}\left(u ; \mathcal{I}_{m}(j)\right) \operatorname{var}_{\frac{1}{\gamma-\varepsilon}}\left(L^{H} ; \mathcal{I}_{m}(j)\right)\right)^{p} \\
& +\delta^{p} m^{-1+p H} \sum_{j=1}^{\lfloor m t\rfloor}\left(\operatorname{var}_{\frac{1}{\gamma-\varepsilon}}\left(L^{H} ; \mathcal{I}_{m}(j)\right)\right)^{p}
\end{aligned}
$$

For the first term we use the Hölder-continuity of the paths of the process $L^{H}$ to show that for all $\varepsilon>0$ such that $-1+p \varepsilon+\frac{p}{\alpha}<0$ it holds almost surely

$m^{-1+p H}\left(\operatorname{var}_{\frac{1}{\gamma-\varepsilon}}\left(L^{H} ; \mathcal{I}_{m}(j)\right)\right)^{p} \leq m^{-1+p H}\left\|L^{H}\right\|_{\gamma-\varepsilon}^{p} m^{p(\varepsilon-\gamma)}=m^{-1+p \varepsilon+\frac{p}{\alpha}}\left\|L^{H}\right\|_{\gamma-\varepsilon}^{p} \stackrel{m \rightarrow \infty}{\rightarrow} 0$.

The selection of $\varepsilon$ with the condition above is possible because $p<\alpha$. For the second summand, we want to show that

$$
\lim _{m \rightarrow \infty} m^{-1+p H} \sum_{j=1}^{\lfloor m t\rfloor}\left(\operatorname{var}_{\frac{1}{\gamma-\varepsilon}}\left(L^{H} ; \mathcal{I}_{m}(j)\right)\right)^{p}<\infty \quad \text { in } L^{1} .
$$

Then we can take the limit for $\delta \rightarrow 0$ which finishes the proof. To show (8), we state the following fact:

$$
\sum_{j=1}^{\lfloor m t\rfloor}\left(\operatorname{var}_{\frac{1}{\gamma-\varepsilon}}\left(L^{H} ; \mathcal{I}_{m}(j)\right)\right)^{p} \leq\lfloor m t\rfloor \max _{j=1, \ldots,\lfloor m t\rfloor}\left(\operatorname{var}_{\frac{1}{\gamma-\varepsilon}}\left(L^{H} ; \mathcal{I}_{m}(j)\right)\right)^{p}
$$


This yields

$$
\begin{aligned}
& \mathbb{E}\left[\lim _{m \rightarrow \infty} m^{-1+p H} \sum_{j=1}^{\lfloor m t\rfloor}\left(\operatorname{var}_{\frac{1}{\gamma-\varepsilon}}\left(L^{H} ; \mathcal{I}_{m}(j)\right)\right)^{p}\right] \\
\leq & \mathbb{E}\left[\lim _{m \rightarrow \infty} m^{-1+p H}\lfloor m t\rfloor \max _{j=1, \ldots,\lfloor m t\rfloor}\left(\operatorname{var}_{\frac{1}{\gamma-\varepsilon}}\left(L^{H} ; \mathcal{I}_{m}(j)\right)\right)^{p}\right] \\
= & \mathbb{E}\left[\lim _{m \rightarrow \infty} m^{-1+p H}\lfloor m t\rfloor\left(\operatorname{var}_{\frac{1}{\gamma-\varepsilon}}\left(L^{H} ; \mathcal{I}_{m}(1)\right)\right)^{p}\right] \\
\leq & T \mathbb{E}\left[\lim _{m \rightarrow \infty} m^{p(H-\gamma+\varepsilon)}\left\|L^{H}\right\|_{\gamma-\varepsilon ;\left[0, \frac{1}{m}\right]}^{p}\right] \\
= & T \mathbb{E}\left[\|X\|_{\gamma-\varepsilon ;[0,1]}^{p},\right.
\end{aligned}
$$

where the last equation is an application of Proposition 2 and $X$ is the linear fractional stable motion, introduced in Example 1. Before finishing the proof we need to gather some information about the linear fractional stable motion. To this end we recall the definition

$$
\|X\|_{\gamma-\varepsilon ;[a, b]}:=\sup _{a \leq s<t \leq b} \frac{\left|X_{t}-X_{s}\right|}{|t-s|^{\gamma-\varepsilon}},
$$

which is finite because of the Hölder-continuity of linear fractional stable motions. Because of the definition of the supremum there are sequences $\left(s_{n}\right)_{n \in \mathbb{N}}$ and $\left(t_{n}\right)_{n \in \mathbb{N}}$, such that $s_{n}, t_{n} \in$ $[0,1] \forall n \in \mathbb{N}$ and

$$
\lim _{n \rightarrow \infty} \frac{\left|X_{t_{n}}-X_{s_{n}}\right|}{\left|t_{n}-s_{n}\right|^{\gamma-\varepsilon}}=\|X\|_{\gamma-\varepsilon ;[0,1]} .
$$

From the self-similarity of the process $X$ we can conclude, that

$$
\frac{\left|X_{t}-X_{S}\right|}{|t-s|^{\gamma-\varepsilon}} \stackrel{\mathcal{D}}{=}|t-s|^{\frac{1}{\alpha}+\varepsilon}\left|X_{1}\right| .
$$

Now we can finish the proof of (8) as follows:

$$
\begin{array}{ll} 
& \mathbb{E}\left[\|X\|_{\gamma-\varepsilon ;[0,1]}^{p}\right] \\
\stackrel{(10)}{=} & \mathbb{E}\left[\left(\lim _{n \rightarrow \infty} \frac{\left|X_{t_{n}}-X_{s_{n}}\right|}{\left|t_{n}-s_{n}\right|^{\gamma-\varepsilon}}\right)^{p}\right] \\
\stackrel{(*)}{\leq} & \lim _{n \rightarrow \infty} \mathbb{E}\left[\left(\frac{\left|X_{t_{n}}-X_{s_{n}}\right|}{\left|t_{n}-s_{n}\right|^{\gamma-\varepsilon}}\right)^{p}\right] \\
\stackrel{(11)}{=} & \lim _{n \rightarrow \infty}\left|t_{n}-s_{n}\right|^{p\left(\frac{1}{\alpha}+\varepsilon\right)} \mathbb{E}\left[\left|X_{1}\right|^{p}\right] \\
\leq & \mathbb{E}\left[\left|X_{1}\right|^{p}\right]<\infty,
\end{array}
$$

where equation (*) follows by the Fatou's lemma. Finally, to complete the proof of $\left\|F_{m}\right\|_{\infty} \rightarrow 0$, we take the limit for $\delta \rightarrow 0$ as mentioned above. 
Now to complete the proof, we have to consider the case $p>1$. As in [CNW06] we use Minkowski's inequality to obtain

$$
\begin{aligned}
& \left|\left(m^{-1+p H} V_{p}^{m}(Z)_{t}\right)^{\frac{1}{p}}-\left(c_{p} \int_{0}^{t}\left|u_{s}\right|^{p} d s\right)^{\frac{1}{p}}\right| \\
\leq & m^{-1+p H}\left(\sum_{j=1}^{\lfloor m t\rfloor} \int_{(j-1) / m}^{j / m} u_{s} d L_{s}^{H}-\left.u_{\frac{j-1}{m}}\left(L_{\frac{j}{m}}^{H}-L_{\frac{j-1}{m}}^{H}\right)\right|^{p}\right)^{\frac{1}{p}} \\
+ & \left|m^{-1+p H}\left(\sum_{i=1}^{\lfloor n t\rfloor} \sum_{j \in I_{n}(i)}^{m}\left|\left(u_{\frac{j-1}{m}}-u_{\frac{i-1}{m}}\right)\left(L_{\frac{j}{m}}^{H}-L_{\frac{j-1}{m}}^{H}\right)\right|^{p}\right)^{\frac{1}{p}}\left(\sum_{i=1}^{\lfloor n t\rfloor}\left|u_{\frac{i-1}{n}}\right|^{p} \sum_{j \in I_{n}(i)}^{m}\left|L_{\frac{j}{m}}^{H}-L_{\frac{j-1}{m}}^{H}\right|^{p}\right)^{\frac{1}{p}}-\left(c_{p} n^{-1} \sum_{i=1}^{\lfloor n t\rfloor}\left|u_{\frac{i-1}{n}}\right|^{p}\right)^{\frac{1}{p}}\right| \\
+ & c_{p}^{\frac{1}{p}}\left|\left(n^{-1} \sum_{i=1}^{\lfloor n t\rfloor}\left|u_{\frac{i-1}{n}}\right|^{p}\right)^{\frac{1}{p}}-\left(\int_{0}^{t}\left|u_{s}\right|^{p} d s\right)^{\frac{1}{p}}\right|
\end{aligned}
$$

and we now proceed similar to the case $p \leq 1$.

Acknowledgements. The financial support of the Deutsche Forschungsgemeinschaft (SFB 823: Statistical modelling of nonlinear dynamic processes, project C5) is gratefully acknowledged.

\section{REFERENCES}

[BCI04] A. Benassi, S. Cohen, and J. Istas. On roughness indices for fractional fields. Bernoulli 10(2), pages 357-373, 2004.

[BNS02] O. E. Barndorff-Nielsen and N. Shephard. Econometric analysis of realized volatility and its use in estimating stochastic volatility models. J. Roy. Statist. Soc. Ser. B, 64(2):253-280, 2002.

[BNS03] O. E. Barndorff-Nielsen and N. Shephard. Realized power variation and stochastic volatility models. Bernoulli, 9(2):243-265, 2003.

[BNS04a] O. E. Barndorff-Nielsen and N. Shephard. Econometric analysis of realized covariation: high frequency based covariance, regression, and correlation in financial economics. Econometrica, 72(3):885-925, 2004.

[BNS04b] O. E. Barndorff-Nielsen and N. Shephard. Power and bipower variation with stochastic volatility and jumps (with discussion). J. Fin. Econometrics, 2(1):1-48, 2004.

[CNW06] J.M. Corcuera, D. Nualart, and J.H.C. Woerner. Power variation of some integral fractional processes. Bernoulli, 12(4):713-735, 2006.

[EW13] S. Engelke and J.H.C. Woerner. A unifying approach to fractional Lévy processes. Stoch. Dyn. 13, 1250017, 2013.

[Kal10] O. Kallenberg. Foundations of Modern Probability. Probability and Its Applications. Springer, 2010.

[Mar06] T. Marquard. Fractional Lévy processes with an application to long memory moving average processes. Bernoulli, 12(6):1099-1126, 2006.

[ST00] G. Samorodnitsky and S. Taqqu. Stable non-gaussian random processes. Stochastic Modeling. Chapman and Hall/CRC, 2000. 
[You36] L.C. Young. An inequality of the Hölder type, connected with Stieltjes integration. Acta Mathematica, 67(1):251-282, 1936.

TU Dortmund, FakUltät FÜr Mathematik, Lehrstuhl IV, 44221 Dortmund, Germany.

E-mail address: sglaser@mathematik. uni-dortmund. de 


